This item was submitted to Loughborough's Research Repository by the author.

Items in Figshare are protected by copyright, with all rights reserved, unless otherwise indicated.

\title{
Motion trapping structures in the three-dimensional water-wave problem
}

PLEASE CITE THE PUBLISHED VERSION

LICENCE

CC BY-NC-ND 4.0

\section{REPOSITORY RECORD}

Mclver, P., and M. Mclver. 2019. "Motion Trapping Structures in the Three-dimensional Water-wave Problem". figshare. https://hdl.handle.net/2134/479. 


\title{
Motion trapping structures in the three-dimensional water-wave problem
}

\author{
P. McIver and M. McIver \\ Department of Mathematical Sciences, Loughborough University, \\ Loughborough, Leics, LE11 3TU, UK
}

Nick Newman has made many important contributions to the theory of the interaction between water waves and structures, and his panel code WAMIT has been adopted as one of the industry standards for the calculation of wave loading on offshore structures. It gives us great pleasure to celebrate Nick's achievements through the presentation of this work on a new type of structure that both draws on his theoretical work and uses WAMIT to perform relevant computations.

\begin{abstract}
Trapped modes in the linearized water-wave problem are free oscillations of finite energy in an unbounded fluid with a free surface. It has been known for some time that such modes are supported by certain structures when held fixed, but recently it has been demonstrated that in two dimensions trapped modes are also possible for freely-floating structures that are able to respond to the hydrodynamic forces acting upon them. For a freely-floating structure such a mode is a coupled oscillation of the fluid and the structure that, in the absence of viscosity, persists for all time. Here previous work on the two-dimensional problem is extended to give motion trapping structures in the three-dimensional water-wave problem that have a vertical axis of symmetry.
\end{abstract}

\section{Introduction}

Within the linearized theory of water waves, certain structures when held fixed can support a trapped mode of a particular frequency [1]. Such a mode is a free oscillation of an unbounded fluid with a free surface that has finite energy, does not radiate waves to infinity, and in the absence of viscosity will persist for all time. The mathematical significance of such a mode is that if, for a specified frequency of fluid oscillation, the structure does not support a trapped mode, then the solutions to the frequency-domain radiation and scattering problems at that frequency are unique. It has been shown recently [2] that trapped modes supported by a fixed structure cannot be excited when that structure is allowed to float freely and is thus able to respond to the hydrodynamic forces acting upon it. In particular, in the time domain a mode cannot be excited by any incident wave or by giving the structure an initial displacement and/or velocity. The realization that modes trapped by fixed structures have no direct relevance to the motion of a freely-floating structure stimulated a search for structures that are able to support trapped modes when allowed to float freely, and has resulted in the discovery of such structures in the two-dimensional water-wave problem [3]. To distinguish the two types of trapped mode, free oscillations supported by a fixed structure will be referred to as a "sloshing trapped mode" and the corresponding structure as a "sloshing trapping structure", while 
a free oscillation that involves the coupled motion of the fluid and a freely-floating structure will be called a "motion trapped mode" and the corresponding structure a "motion trapping structure". The mathematical significance of a motion trapped mode is that if, for a specified frequency of fluid oscillation, the structure does not support such a mode, then there is a unique solution to a problem in which the structure is able to float freely.

Trapped modes correspond to resonances of the water-wave problem and, at least in all of the examples known at this time, are associated with a restriction of the free surface either by a surface-piercing structure (as in [1]) or by a submerged structure that is very close to the free surface [4]. There have been many studies of structures with an enclosed portion of the free surface, or moonpool, and of particular relevance to the present work is the investigation by Newman [5] of a floating torus with a circular cross section. The hydrodynamic coefficients of the torus display characteristic rapid changes in the neighbourhoods of certain frequencies that are associated with sloshing in the moonpool. Newman [5] also investigated the excitation of a floating torus by incident waves and observed resonant behaviour of the body motions. Simple structures without a moonpool exhibit motion resonances only in heave and at a single frequency. In contrast to this, the torus exhibits many resonances of the body motion and they occur for all modes of motion. The hydrodynamic properties of the motion trapping structures discussed here are very similar to those of the floating torus investigated by Newman [5].

In the terminology of the linearized theory of water waves, the study of motion trapping structures involves the study of a radiation problem in which the structure oscillates in the absence of incident waves. For a structure to support motion trapped modes with a particular frequency $\omega=\omega_{0}$ in a single mode of oscillation, the corresponding frequency-dependent hydrodynamic coefficients must satisfy two conditions at this frequency. First of all the damping coefficient must be zero, so that there is no wave radiation to infinity, and secondly the added mass coefficient must satisfy a resonance condition obtained from the requirement that the equation of motion for the structure has a non-trivial solution in the absence of any forcing. McIver and McIver [3] have shown that there are two-dimensional structures whose hydrodynamic coefficients satisfy these conditions for motions in both the heave and surge directions. They used an inverse procedure similar

to that used by Kyozuka and Yoshida [6] to obtain wave-free oscillating structures, and by McIver [1] to obtain sloshing trapping structures.

Here the construction used by McIver and McIver [3] is extended to three dimensions and, in particular, it is shown that motion trapped modes may be supported by particular heaving structures with a vertical axis of symmetry. The problem is formulated and the conditions for resonance obtained in $\S 2$. Specific constructions of motion trapping structures in are described in $\S 3$ and some consequences of the existence of motion trapping structures are discussed in $\S 4$.

\section{Formulation}

Cartesian coordinates $x, y, z$ are chosen with $z$ directed vertically upwards from the mean free surface. In addition, $R, \theta$ will be used to denote spherical coordinates with $\theta$ measured from the downward vertical and a cylindrical polar coordinate $r=R \sin \theta$ will be employed to measure horizontal distance from the $z$ axis.

Consider a surface-piercing structure constrained to move freely in the vertical direction with initial displacement $Z(0)$ and initial velocity $\dot{Z}(0)$. In the absence of any moorings, the Fourier transform of the time-domain equation of motion yields the frequency-domain equation of motion

$$
\left[\rho g W-\omega^{2}\{M+a(\omega)+\mathrm{i} b(\omega) / \omega\}\right] v(\omega)=-\mathrm{i} \omega[X(\omega)+M \dot{Z}(0)]-\rho g W Z(0)
$$

(see, for example, McIver [2]; the Fourier transform is defined so that frequency-domain motions are equiv- 
alent to oscillations with a time dependence $\mathrm{e}^{-\mathrm{i} \omega t}$ ). Here $\rho$ is the fluid density, $g$ is the acceleration due to gravity, $W$ is the water plane area, $M$ is the mass of the structure (which by Archimedes principle is $\rho$ times the submerged volume $V$ ), $a$ is the added mass coefficient, $b$ is the damping coefficient, $v$ is the (complex) amplitude of the structural velocity, and $X$ is the exciting force due to wave incidence on a fixed structure. Note that the functions of frequency in equation (1) are Fourier transforms in time of physical quantities so that, for example, $v(\omega)$ has the dimensions of length rather than velocity.

Given the hydrodynamic coefficients at a particular frequency $\omega$, there will be a unique solution of the equation of motion (1) for the velocity $v(\omega)$ provided that the homogeneous equation

$$
\left[\rho g W-\omega^{2}\{M+a(\omega)+\mathrm{i} b(\omega) / \omega\}\right] v=0
$$

has only the trivial solution $v=0$. Necessary conditions for the existence of a non-zero $v$, corresponding to a motion trapped mode, for some particular frequency $\omega=\omega_{0}$ are that

$$
\rho g W-\omega_{0}^{2}\left\{M+a\left(\omega_{0}\right)\right\}=0
$$

and

$$
b\left(\omega_{0}\right)=0 .
$$

The second of these conditions implies that, when forced to oscillate, the structure does not radiate waves to infinity.

Kyozuka \& Yoshida [6] constructed wave-free structures with $b\left(\omega_{0}\right)=0$ by an inverse procedure in which the velocity potential for the fluid motion is expressed in terms of singular solutions of the governing equations, and then the surface of a structure is obtained from examination of the streamlines of a modified potential. In general, a structure obtained in this way does not possess hydrodynamic characteristics that also allow equation (3) to be satisfied at the same frequency $\omega=\omega_{0}$. However, an application of Green's theorem shows how this can be achieved for particular classes of structure.

Denote by $\phi_{0}$ the velocity potential corresponding to the vertical oscillations of a wave-free structure at frequency $\omega=\omega_{0}$ so that, in particular,

$$
\frac{\partial \phi_{0}}{\partial n}=n_{z} \quad \text { on } \quad \Gamma
$$

where $n$ is a normal coordinate to the wetted surface of the structure $\Gamma$, directed out of the fluid, and $n_{z}$ is the vertical component of the unit normal to $\Gamma$. By definition, the added mass

$$
a\left(\omega_{0}\right)=\rho \iint_{\Gamma} \phi_{0} n_{z} \mathrm{~d} S
$$

where the fact that $b\left(\omega_{0}\right)=0$ has been used. For vertically axisymmetric three-dimensional motion in fluid of infinite depth any wave-free potential must satisfy

$$
\phi_{0}=\frac{\mu \cos \theta}{R^{2}}+o\left(\frac{1}{R^{2}}\right) \quad \text { as } \quad R \rightarrow \infty
$$

where $\mu$ is a constant, so that to leading order $\phi_{0}$ is dipole-like at infinity (see [7]); it is possible that $\mu$ may be zero.

Let $S$ denote the union of $\Gamma$ with the free surface $F$ and a closing hemisphere $S_{\infty}$ at infinity in $z<0$. Application of Green's theorem over $S$ to the harmonic functions $\phi_{0}$ and

$$
u=z+1 / K
$$

yields

$$
\iint_{S}\left[\phi_{0} \frac{\partial u}{\partial n}-u \frac{\partial \phi_{0}}{\partial n}\right] \mathrm{d} S=0
$$


The contribution to the integral from $F$ is zero, as both $\phi_{0}$ and $u$ satisfy the free-surface condition, and it follows from the asymptotic form (7) that

$$
\iint_{S_{\infty}}\left[\phi_{0} \frac{\partial u}{\partial n}-u \frac{\partial \phi_{0}}{\partial n}\right] \mathrm{d} S=-2 \pi \mu
$$

From the boundary condition (5),

$$
\iint_{\Gamma}\left[\phi_{0} \frac{\partial u}{\partial n}-u \frac{\partial \phi_{0}}{\partial n}\right] \mathrm{d} S=\iint_{\Gamma}\left[\phi_{0} n_{z}-\left(z+\frac{1}{K}\right) n_{z}\right] \mathrm{d} S
$$

and the integral of $\phi_{0}$ may be written in terms of the added mass using (6). The divergence theorem may be used to show that for an arbitrary $\chi$

$$
\iint_{\Gamma+W} \chi n_{z} \mathrm{~d} S=-\iiint_{D} \nabla \chi \cdot \mathbf{e}_{z} \mathrm{~d} V
$$

where $D$ is the submerged region occupied by the structure, $\mathbf{e}_{z}$ is a unit vector in the $z$ direction, and the normal coordinate $n$ is directed into $D$. The choice $\chi=z$ then gives

$$
\iint_{\Gamma} z n_{z} \mathrm{~d} S=-V=-M / \rho
$$

(because $z=0$ on $W$ ), and the choice $\chi=1$ yields

$$
\iint_{\Gamma} n_{z} \mathrm{~d} S=-\iint_{W} n_{z} \mathrm{~d} S=W
$$

(because $n_{z}=-1$ on $W$ ). Thus, the application of Green's theorem results in

$$
\rho g W-\omega_{0}^{2}\left\{M+a\left(\omega_{0}\right)\right\}=-2 \pi \mu \rho \omega_{0}^{2}
$$

so that the resonance condition (15) may be satisfied if and only if the far-field dipole coefficient $\mu$ is zero.

\section{Construction of motion trapping structures}

Kyozuka \& Yoshida [6] used an inverse method to obtain structures with zero radiation damping by looking for suitable streamlines of the flows generated by singular wave-free potentials. Here the same method is adopted with the additional requirement that the wave-free potential $\phi_{0}$ has a far-field dipole coefficient $\mu=0$. From equation (5), if a suitable structure could be identified, the modified potential

$$
\phi=z-\phi_{0}
$$

would satisfy

$$
\frac{\partial \phi}{\partial n}=0 \quad \text { on } \quad \Gamma
$$

so that $\Gamma$ is a stream surface of the flow corresponding to $\phi$. Hence motion trapping structures are obtained from any stream surface of this flow that isolate the singularities of $\phi$ from infinity.

In the two-dimensional case reported elsewhere [3], the wave-free potential was formed from two wave sources placed at points in the free surface chosen to eliminate wave radiation to infinity, combined with standard wave free potentials that have a dipole component. In three dimensions the construction is based on equivalent solutions that are singular on a circular ring of radius $c$ in the free surface. By analogy with 
the two-dimensional case, a wave-free ring source $\phi_{\mathrm{s}}$ and a potential $\phi_{\mathrm{d}}$ that is dipole-like at infinity are used to give a total wave-free potential in the form

$$
\phi_{0}=\delta\left(\phi_{\mathrm{s}}+\sigma \phi_{\mathrm{d}}\right)
$$

where $\delta$ is a free parameter and $\sigma$ is chosen to ensure that $\phi_{0}$ has no dipole component in the far field.

Provided that $K c=j_{0 n}$, where $j_{0 n}$ denotes the $n$th zero of the Bessel function $J_{0}$, a wave-free ring-source potential $[8]$ is

$$
\phi_{\mathrm{s}}=8 c \int_{0}^{\infty}(\mu \cos \mu z+K \sin \mu z) I_{0}\left(\mu r_{<}\right) K_{0}\left(\mu r_{>}\right) \frac{\mu \mathrm{d} \mu}{\mu^{2}+K^{2}},
$$

where $I_{0}$ and $K_{0}$ are modified Bessel functions,

$$
r_{<}=\min \{r, c\} \quad \text { and } \quad r_{>}=\max \{r, c\} .
$$

The Stokes stream function corresponding to $\phi_{\mathrm{s}}$ is

$$
\psi_{\mathbf{s}}=\left\{\begin{array}{cl}
8 K c r \int_{0}^{\infty}(\mu \sin \mu z-K \cos \mu z) I_{1}(\mu r) K_{0}(\mu c) \frac{\mu \mathrm{d} \mu}{\mu^{2}+K^{2}}, & 0 \leq r<c, \\
-8 K c r \int_{0}^{\infty}(\mu \sin \mu z-K \cos \mu z) I_{0}(\mu r) K_{1}(\mu c) \frac{\mu \mathrm{d} \mu}{\mu^{2}+K^{2}}, & r>c,
\end{array}\right.
$$

(see [10] where the numerical evaluation of $\psi_{\mathrm{s}}$ is also discussed).

For the wave-free dipole $\phi_{\mathrm{d}}$ it is convenient to work in terms of toroidal coordinates $\alpha$ and $\beta$ related to $r$ and $z$ by

$$
r=\frac{c \sinh \alpha}{\cosh \alpha-\cos \beta} \quad \text { and } \quad z=-\frac{c \sin \beta}{\cosh \alpha-\cos \beta}, \quad 0 \leq \alpha<\infty, \quad 0 \leq \beta \leq \pi
$$

(see, for example, Lebedev [9]). On the inside of the singular ring the free surface is $\beta=\pi$ and outside the ring it is $\beta=0$; the ring itself corresponds to the limit $\alpha \rightarrow \infty$. C. M. Linton (private communication) has derived a set of wave-free potentials that are singular on a ring in the free surface and are dipole-like in the far field. The one that will be used here is

$$
\begin{aligned}
\phi_{\mathrm{d}}=(\cosh \alpha-\cos \beta)^{1 / 2}\left\{K c P_{\frac{1}{2}}(\cosh \alpha) \sin \beta+P_{\frac{1}{2}}(\cosh \alpha)\right. & \cos \beta \\
& \left.-\frac{1}{4} P_{-\frac{1}{2}}(\cosh \alpha)-\frac{3}{4} P_{\frac{3}{2}}(\cosh \alpha) \cos 2 \beta\right\},
\end{aligned}
$$

where $P_{\nu}$ denotes an associated Legendre function. This particular potential is chosen because

$$
\phi_{\mathrm{d}} \sim \frac{C_{2} \cos 2 \theta}{R^{2}} \text { as } R \rightarrow 0,
$$

for a non-zero constant $C_{2}$, which mimics the equivalent behaviour in the two-dimensional problem [3]. The Stokes stream function corresponding to $\phi_{\mathrm{d}}$ is

$$
\begin{aligned}
\psi_{\mathrm{d}}=c(\cosh \alpha-\cos \beta)^{1 / 2}\left\{K c \left[H(\alpha, \beta) P_{\frac{1}{2}}(\cosh \alpha)-\right.\right. & \left.P_{-\frac{1}{2}}(\cosh \alpha)\right] \\
& \left.+\frac{3}{2} \sin \beta\left[H(\alpha, \beta) P_{\frac{3}{2}}(\cosh \alpha)-P_{\frac{1}{2}}(\cosh \alpha)\right]\right\}
\end{aligned}
$$

where

$$
H(\alpha, \beta)=\frac{1-\cosh \alpha \cos \beta}{\cosh \alpha-\cos \beta}
$$




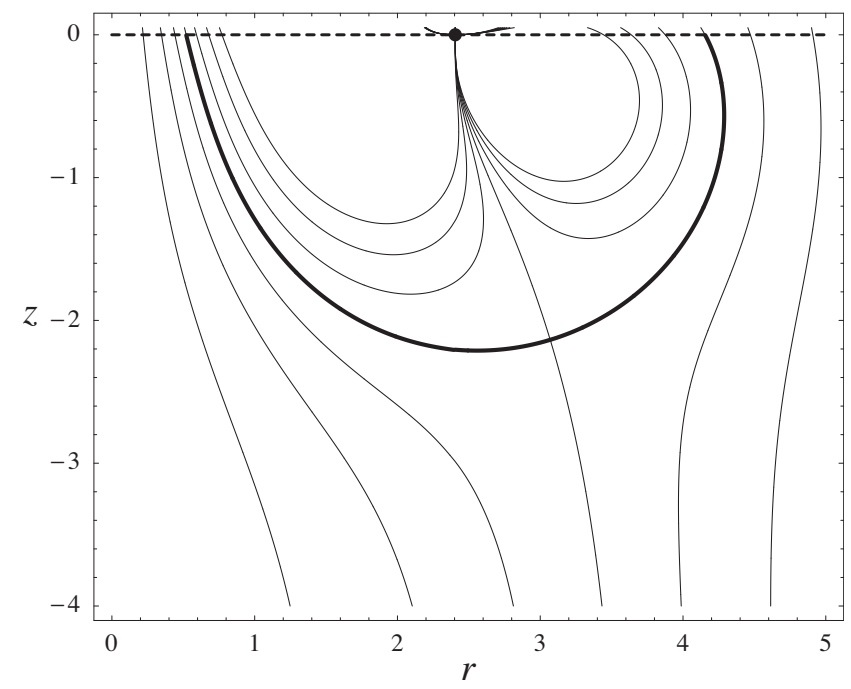

Figure 1: Cross section of the stream surfaces corresponding to the Stokes stream function given by (27); $K=1, c=j_{01}, \delta=1$.

The required stream surfaces are the level surfaces of

$$
\psi=-x-\delta\left(\psi_{\mathrm{s}}+\sigma \psi_{\mathrm{d}}\right) .
$$

Integration by parts shows that on $r=0$,

$$
\phi_{\mathrm{s}} \sim-\frac{4 \pi c}{K z^{2}} \quad \text { as } \quad z \rightarrow-\infty
$$

and, from the definitions of the toroidal coordinates in equation (22),

$$
\phi_{\mathrm{d}} \sim \frac{2^{3 / 2} K c^{3}}{z^{2}} \quad \text { as } \quad z \rightarrow-\infty
$$

so that the choice $\sigma=\sqrt{2} \pi /\left(K^{2} c^{2}\right)$ ensures that $\phi_{0}$ has no far-field dipole component.

First of all the choice $K c=j_{01} \approx 2.40$ is made and a typical stream surface pattern is shown in figure 1 . There is a dividing stream surface that, in $z<0$, separates the flow connected to large depths from that entering the singularity. Part of this stream surface, shown as a thick line in the figure, corresponds to the surface of a motion trapping structure. For a given $\phi_{0}$ there is a single suitable stream surface that isolates the singular ring in a suitable way. However, as the parameter $\delta$ is varied a family of motion trapping structures is obtained and some examples are shown in figure 2. A perspective view of one of these structures in figure 3 and there is a superficial resemblance to the sloshing trapping structures obtained by McIver and McIver [10]. Numerical calculations suggest that motion trapping structures may be obtained for any positive value of $\delta$.

For $K c=j_{02} \approx 5.52$ the flow field shows some extra structure as can be seen from the example shown in figure 4. Again there is a single dividing stream surface and the surface of a motion trapping structure is given by that part of the surface around the singularity. However, in addition to the single element trapping structure obtained from the dividing streamline, there is a family of structures with two concentric elements the innermost of which is formed from any stream surface adjacent to $r=0$ that has two ends on the free surface. As for the case $K c=j_{01}$, further families of trapping structures may be obtained through variation of $\delta$. 


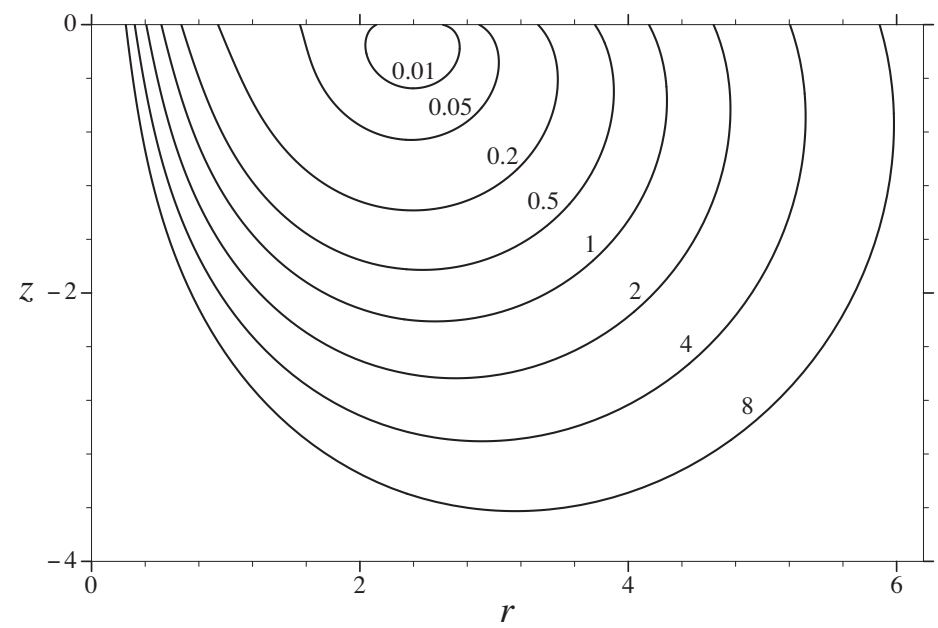

Figure 2: Cross section of the stream surfaces corresponding to the Stokes stream function given by (27); $K=1, c=j_{01}$. Each structural surface is marked with the corresponding value of $\delta$.

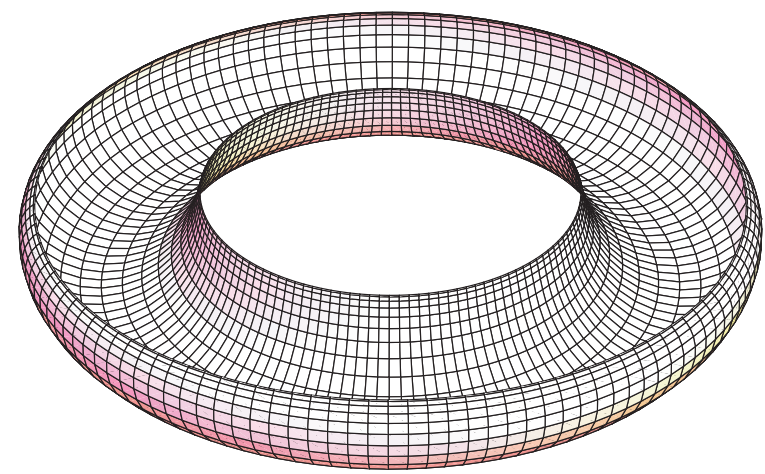

Figure 3: The submerged surface of the trapping structure corresponding to $\delta=0.05$ in figure 2 .

\section{Consequences of the existence of motion trapped modes}

Newman [11] used the panel code WAMIT to perform detailed numerical calculations of the frequency-domain hydrodynamic coefficients for vertically axisymmetric sloshing trapping structures and found near-singular behaviour around the trapped-mode frequency for the added mass and damping coefficients and for the exciting force. This behaviour arises because the frequency-domain radiation potential has a pole at the trapped-mode frequency although, of course, a numerical method cannot capture this exactly. Furthermore, in the exact solution to a scattering problem, the scattering potential is orthogonal to the trapped mode and hence well-behaved at the trapped-mode frequency [12]. In the case of a motion trapping structure all of the hydrodynamic coefficients are well behaved at the resonant frequency $\omega=\omega_{0}$ and as, by construction, the damping coefficient $b\left(\omega_{0}\right)=0$ it follows from the Haskind relation that the exciting force $X\left(\omega_{0}\right)=0$.

Singular behaviour in a problem involving a motion trapping structure can arise through the solution of the equation of motion (1) for $v(\omega)$. If one or both of the initial displacement and velocity, $Z(0)$ and $\dot{Z}(0)$ respectively, are non zero then from (1) the velocity $v$ will have a simple pole at $\omega=\omega_{0}$. Thus, in these circumstances, the solution to the boundary-value problem will not exist at the resonant frequency. However, 


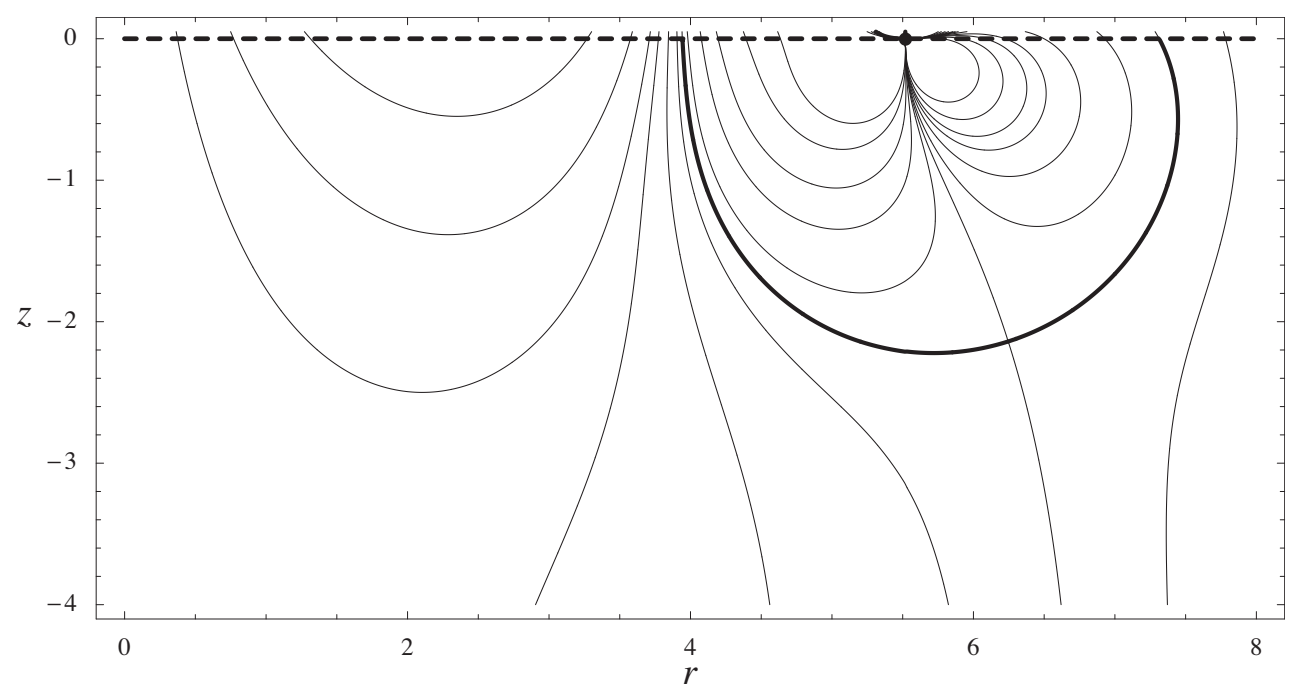

Figure 4: Cross section of the stream surfaces corresponding to the Stokes stream function given by (27); $K=1, c=j_{02}, \delta=1$.

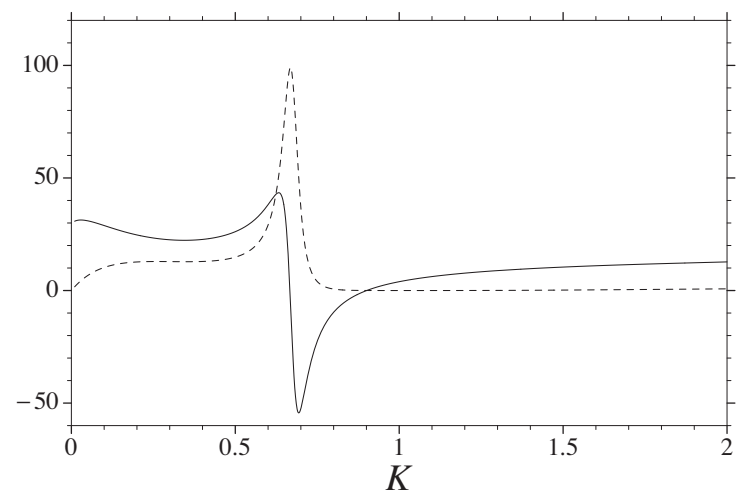

Figure 5: Heave added mass $a / \rho(-)$ and damping $b / \rho \omega(---)$ coefficients for the structure shown in figure 3 for which a motion trapped mode exists at $K=1$.

if $Z(0)=\dot{Z}(0)=0$ then the analytic nature of $X(\omega)$ means that the zero in $X$ annuls the zero arising from (3) and (4) and $v(\omega)$ is non-singular at the resonant frequency so that the solution to the boundary-value problem exists, although it will not be unique because any multiple of the motion trapped mode can be added to the solution. In numerical calculations of motion trapping structures this behaviour will not be captured precisely (the exciting force and damping coefficient will be small but non zero and the resonance condition will not be satisfied exactly) and hence there will be a pole of $v(\omega)$ close to the positive real $\omega$ axis in the lower half of the complex frequency domain. (Newman [5] analyzed the motion of a floating torus with a circular cross section and found resonances of this type; he also pointed out that when the resonance condition (3) is not satisfied a zero in $X(\omega)$ will be accompanied by a zero in $v(\omega)$ at the same frequency.) With this type of complex resonance the hydrodynamic coefficients are well behaved in the vicinity of $\omega=\omega_{0}$ and the velocity of the structure undergoes very rapid changes as a function of frequency. For the particular motion trapping structure given in figure 3 , the added mass and damping coefficients are shown as a function 

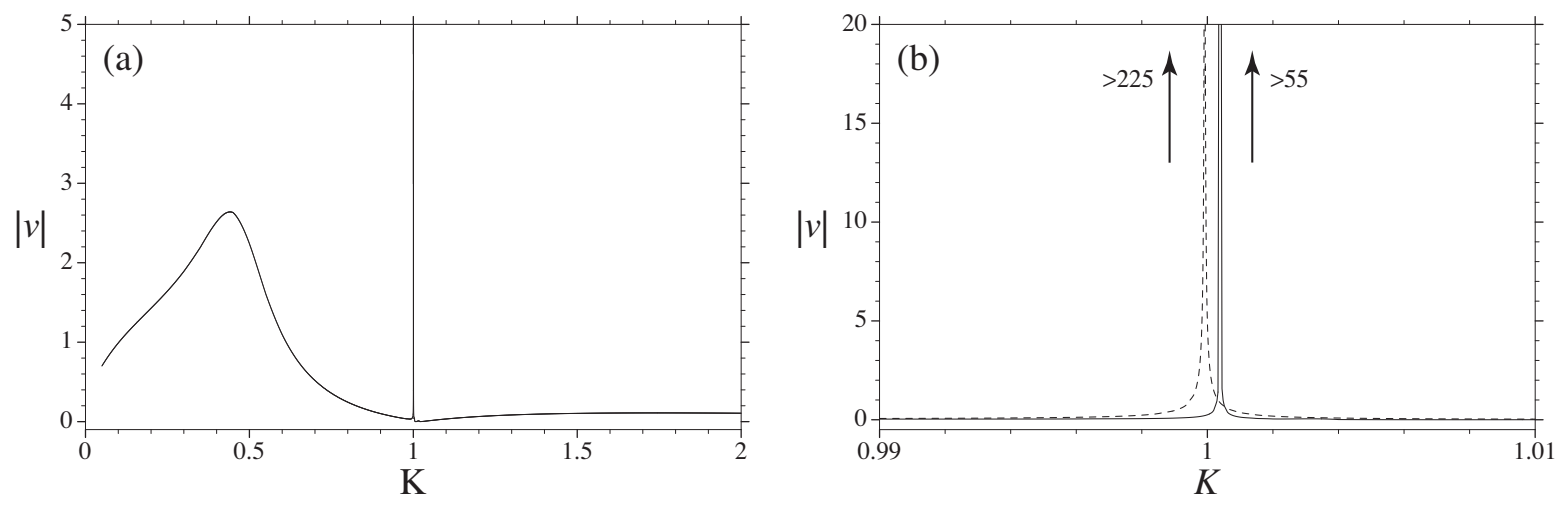

Figure 6: Vertical velocity $v$ excited by an incident wave of unit amplitude for the structure shown in figure 3 for which a motion trapped mode exists at $K=1$. In (b) comparison is made between calculations with $8704(---)$ and $2176(-)$ panels on a quarter of the wetted surface (additional free-surface panels were used to eliminate irregular frequencies).

of frequency in figure 5 (the computations were performed using version 5.4 of the panel code WAMIT). The rapid variations in added mass and damping associated with sloshing in the moonpool occur well away from the motion resonance at $K=1$. It is typical of the motion trapping structures generated by the present method that the damping coefficient is very close to zero over quite a wide range of frequencies around the resonant frequency. In this example the added mass is positive at the resonant frequency, but it is a simple matter to generate examples for which the added mass is negative at resonance.

Figure 6 shows the vertical velocity as a function of frequency when the structure is excited by incident waves of unit amplitude. As observed by Newman [5] for a floating torus, there are many motion resonances and two of these can be seen in figure 6(a). The "spike" arising from what, in this numerical calculation, is only a close approximation to a motion trapping structure is clearly evident near $K=1$. The computations were performed with two different levels of discretization and figure 6(b) shows that the spike in the velocity is discernible only in a very narrow band which could easily be missed, and the spike moves closer to $K=1$ as the number of panels on the wetted surface of the structure is increased. It is very difficult to obtain reliable computations near the resonant frequency because, when solving the equation of motion for $v(\omega)$, it is necessary to evaluate the ratio of two quantities that are both very close to zero.

McIver and McIver [3] have examined the behaviour of two-dimensional motion trapping structures in the time domain. They found that if such a structure is initially at rest in its equilibrium position then the motion trapped mode cannot be excited by an incident wave packet. This is a direct consequence of the zero in the exciting force at $\omega=\omega_{0}$. However, if the structure is given an initial non-zero displacement or velocity then the mode will be excited so that after an initial transient has died away the structure and fluid motions settle to oscillations at the trapped-mode frequency. Similar conclusions apply to the three-dimensional structures obtained here.

\section{Conclusion}

It has been shown here that there exists three-dimensional structures with a vertical axis of symmetry that support heave motion trapped modes. Thus, for a particular frequency, there is a coupled oscillation of the structure and fluid that does not radiate waves to infinity and which, in the absence of viscosity, will persist 
for all time. This extends previous work on motion trapped modes in two dimensions. Further work might include the construction of non-axisymmetric structures similar to the sloshing trapping structures found by McIver and Newman [13], and surge motion trapping structures as found in the two dimensional problem by McIver and McIver [3].

\section{Acknowledgement}

The authors are grateful to Professor C. M. Linton for giving access to his notes on "toroidal" wave-free potentials.

\section{References}

1. M. McIver, An example of non-uniqueness in the two-dimensional linear water wave problem. Journal of Fluid Mechanics 315 (1996) 257-266.

2. P. McIver, Complex resonances in the water-wave problem for a floating structure. Journal of Fluid Mechanics 536 (2005) 423-443.

3. P. McIver and M. McIver, Trapped modes in the water-wave problem for a freely-floating structure. Submitted for publication.

4. M. McIver and R. Porter, Trapping of waves by a submerged elliptical torus. Journal of Fluid Mechanics 456 (2002) 277-293.

5. J. N. Newman, The motions of a floating slender torus. Journal of Fluid Mechanics 83 (1977) 721-735.

6. Y. Kyozuka and K. Yoshida, On wave-free floating body forms in heaving oscillation. Applied Ocean Research 3 (1981) 183-194.

7. A. Hulme, The wave forces acting on a floating hemisphere undergoing forced periodic oscillations. Journal of Fluid Mechanics 121 (1982) 443-463.

8. A. Hulme, A ring-source/integral-equation method for the calculation of hydrodynamic forces exerted on floating bodies of revolution. Journal of Fluid Mechanics, 128 (1983) 387-412.

9. N. N. Lebedev, Special functions and their applications. New York: Dover (1972) 308 pp.

10. P. McIver and M. McIver, Trapped modes in an axisymmetric water-wave problem. Quarterly Journal of Mechanics and Applied Mathematics 50 (1997) 165-178.

11. J. N. Newman, Radiation and diffraction analysis of the McIver toroid. Journal of Engineering Mathematics 35 (1999) 135-147.

12. M. McIver, Resonances in the unbounded water wave problem. In: Proceedings of the 12th International Workshop on Water Waves and Floating Bodies, held in Carret-le-Rouet, France, 16-19 March 1997 $177-180$.

13. P. McIver and J. N. Newman, Trapping structures in the three-dimensional water-wave problem. Journal of Fluid Mechanics 484 (2003) 283-301. 